\title{
Antiemetic prophylaxis with high dose metoclopramide or lorazepam in vomiting induced by chemotherapy
}

Emesis induced by cytotoxic agents is an important side effect that may determine a patient's acceptance of cancer chemotherapy and may be responsible for his defaulting from potentially effective treatment. Despite the availability of several antiemetic drugs, this complication is generally poorly controlled. Gralla et al found metoclopramide in high intravenous doses to be an effective antiemetic agent, better than placebo or prochlorperazine, in chemotherapy with cisplatin. ${ }^{1}$ Maher reported the use of lorazepam in antiemetic premedication for cytotoxic treatment. ${ }^{2}$ We evaluated both these drugs in a crossover study of emesis induced by cisplatin and dacarbazine, two of the most highly emetic cytotoxics.

\section{Patients, methods, and results}

We studied 19 patients with disseminated malignant melanoma (nine men 10 women, median age 52) requiring chemotherapy with the (B)DPV3 regimen. ${ }^{3}$ Only two patients had previously received a course of adjuvant stated a preference for lorazepam because of these properties. In our experience this drug has made possible lengthy treatments with highly emetic cytotoxic agents.

Despite the moderate dose of cisplatin used in this trial, the number of emetic episodes observed with metoclopramide was greater than that observed in an earlier report, ${ }^{1}$ and is in agreement with the number described in a more recent communication. ${ }^{5}$ The study has also highlighted the variable reaction of patients to the side effects of chemotherapy. We think that the final arbiter of the toxicity of cancer treatment should be the patient, who can withdraw from it when it no longer constitutes treatment.

We thank Sister C Russell of Wolfe Barry ward, Sister D Hooper of Rock Carling ward, and their senior nurses. Without their help and cooperation this study would not have been possible. We thank Wyeth Pharma ceuticals and Beecham Research Laboratories for financial support and help in the statistical analysis of the results. Metoclopramide was generously donated by Beecham Research Laboratories for the trial. The study was

Efficacy of antiemetics in 19 patients undergoing chemotherapy

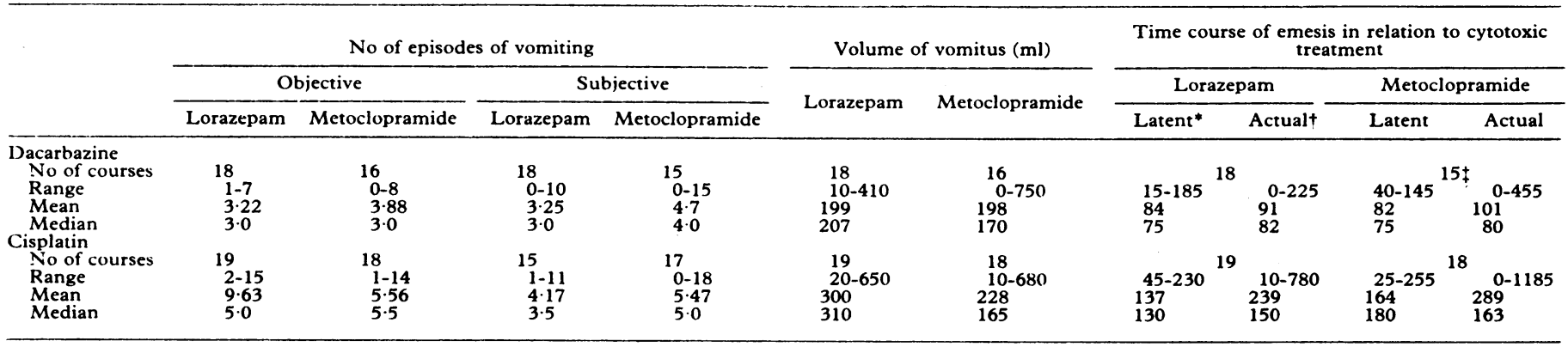

* Latent: period in minutes from time emetic drug was given to first episode of vomiting.

tActual: period in minutes from first to last episode of vomiting.

† One patient in whom vomiting did not occur is not included in this group.

vindesine chemotherapy and neither had experienced nausea or vomiting No patients had cerebral metastases and all had a Karnofsky performance of $>60 \%$.

During the trial cisplatin was given in a standard dose of $60 \mathrm{mg}$ by intravenous bolus injection with mannitol diuresis and hydration on day 1 of the cycle. Vinblastine and bleomycin were also given on day 1 , and vindesine on day 2. Dacarbazine $1 \mathrm{~g}$ by intravenous bolus was given with vincristine on day 14. The cycle was repeated on day 28.

Patients were alternately allocated to receive either high dose metoclopramide or lorazepam during the first of two cytotoxic cycles and the alternative antiemetic for the second cycle. Lorazepam $2.5 \mathrm{mg} / \mathrm{m}^{2}$ was given by slow intravenous injection 30 minutes before the injection of dacarbazine or cisplatin. Metoclopramide was given by the method described by Gralla $e t a^{1}$ in five separate aliquots of $2 \mathrm{mg} / \mathrm{kg}$ each infused over 15 minutes, starting 30 minutes before the emetic cytotoxic agent.

Assessment of antiemetic efficacy was carried out objectively by senior nursing staff, who recorded on appropriate charts the time, volume, and number of episodes of vomiting. Subjective assessment was carried out by each patient 24 hours after the emetic chemotherapy by questionnaire. Statistical evaluation of results was performed using a standard crossover analysis ${ }^{4}$ and Wilcoxon's matched paired sign rank test.

Of the 19 patients, 16 completed two full cycles of chemotherapy and 15 of these had two trials of both high dose metoclopramide and lorazepam. One patient, who had a third trial of lorazepam in preference to metoclopramide, was excluded from the statistical analysis of the dacarbazine arm. The median administered dose of lorazepam was $4 \mathrm{mg}$ (range $3-5 \mathrm{mg}$ ) and of metoclopramide $643 \mathrm{mg}$ (range $380-1070 \mathrm{mg}$ ). During 34 courses of metoclopramide only one dystonic reaction was noted. Sedation, as classified by Gralla $e t$ al, ${ }^{1}$ was mild or absent with metoclopramide but always moderate or marked with lorazepam. Results of antiemetic efficacy are shown in the table.

\section{Comment}

No statistically significant difference has been observed in antiemetic prophylaxis between lorazepam and high dose metoclopramide. Lorazepam proved more valuable, however, with its simple administration and amnesic and anxiolytic effect. On completion of the trial 15 patients supported by the Radiotherapy Research Fund (Westminster Hospital), and the Medical Oncology Research Fund and the Lawson Trust (Westminster Medical School)

1 Gralla RJ, Itri LM, Pisko SE, et al. Antiemetic efficacy of high-dose metoclopramide: randomized trials with placebo and prochlorperazine in patients with chemotherapy-induced nausea and vomiting. $N$ Engl f Med $1981 ; 305: 905-9$

${ }^{2}$ Maher J. Intravenous lorazepam for the prevention of nausea and vomiting associated with cancer chemotherapy. Lancet $1981 ; \mathrm{i}: 91$.

${ }^{3}$ Retsas S, Nicoll J. Effective cyclic combination chemotherapy in advanced metastatic malignant melanoma (MMM), using cis-platin, vinblastine, vindesine, with and without bleomycin, alternating with DTIC and vincristine. The (B)DPV 3 regime. Proceedings of the 20th annual meeting of the American Society of Clinical Oncology. Danbury, Conn: Waverly Press, 1984. Vol 3, 255. Abstract C-998.

' Hills M, Armitage P. The two-period cross-over clinical trial. $\mathrm{Br} \mathcal{f} \mathrm{Clin}$ Pharmacol 1979;8:7-20.

5 Van Hazel GA, Frytak S, Anderson SA, O'Fallon JR, Moertel CG, Long $\mathrm{HJ}$. A double-blind cross-over study comparing high dose metoclopramide $(\mathbf{M})$ to dexamethasone + prochlorperazine $(\mathrm{D}+\mathrm{P})$ as antiemetics during cisplatin chemotherapy (CP). Proceedings of the 19th annual meeting of the American Society of Clinical Oncology. Danbury, Conn: Waverly Press, 1983. Vol 2, 85. Abstract C-329.

(Accepted 11 April 1984)

Department of Radiotherapy and Oncology, Medical Oncology Unit, Westminster Hospital, London SW1P 2AP

$S$ J BOWCOCK, MA, MRCP, senior house officer in medicine

A D STOCKDALE, BSC, MRCP, senior house officer in medicine

J A R BOLTON, MB, BS, preregistration house physician

A A KANG, MB, BS, preregistration house physician

$S$ RETSAS, MD, MRCP, senior lecturer in medical oncology and honorary consultant physician

Correspondence to: Dr S Retsas. 